Short Communication

\title{
ADAPTATION OF AN OCCUPATIONAL STRESS INDEX
}

\author{
Md. Abdul Latif* and Sabina Sultana
}

Department of Psychology, Rajshahi University, Rajshahi 6205, Bangladesh

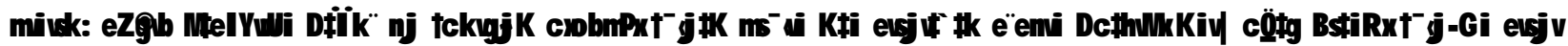

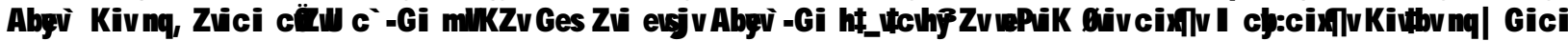

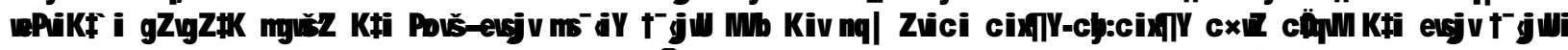

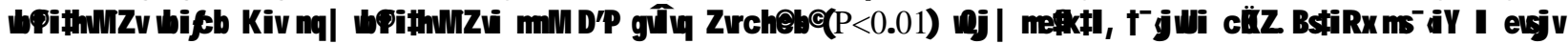

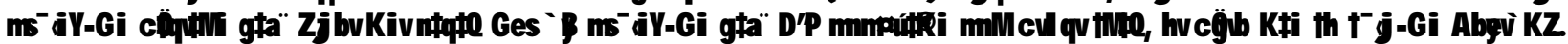

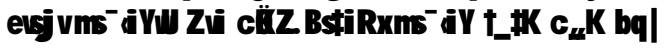

Key words: Occupational stress index (OSI), reliability, test-retest method, correlation of coefficient

Occupational stresses have become predominant feature of modern life. It has far-reaching effects on employees' behaviour and adjustments as well as off the job. For that reason need for systematic study of stress in organizational setting has recently increased dramatically. A considerable portion of organization research involves the study of stress relating to job. Assessment of stress relating to job is important also for personal counselling. Considering the immense importance of assessing occupational stress, the problem of its measurement arises. But a review of the literature reveals the lack of valid and reliable instrument for measuring stress relating job. Although a number of scales measuring stress relating job may be available from other countries, they are unlikely to be appropriate in our culture since the items of these scales were developed to suit their own culture which is different from ours in many respects. Therefore, it has become essential to develop appropriate scale for measuring occupational stress in our country.

Occupation Stress Index (OSI) is a widely acceptable scale for measuring job stress. It has been used effectively by psychologists in researches. This famous scale, however, can not be properly used in our country for two reasons: First, the test is in English and second, some of the items are culturally unfamiliar to the employees of our country. The authors feel that there is a need for translating and adapting the scale to make it suitable for measuring occupational stress of our employees. It was with this end in view that the present study was designed. The aim of the present study was to translate and adapt OSI as a valid and *Corresponding author reliable instrument for measuring values of the employees of our country.

The English version of the 0SI was originally developed by Srivastava and Singh (1984) at Banaras Hindu University, Uttar Pradesh (UP), India. The scale purports to measure the extent of stress which employees perceive from various constituents and conditions of their job. The scale may be administered to the employees of every level operating in context of industries or other non-production organizations. The scale consists of 46 items, comprising 28 'true-keyed' and 18 'false-keyed' and each of which is rated on a five-point scale. The items are related to almost all relevant components of job life which cause stress in some way or other. The account of items constituting various sub-scales along with their indices of internal consistency is shown in Table 1.

The reliability of the scale was measured through splithalf (odd-even) method and the Cronbach's alphacoefficient for the scales as a whole was found to be 0.935 and 0.90 , respectively. The reliability of 12 subscales was also computed through split-half method, and all the sub-scales were found rightly reliable. The validity of the OSI was determined by computing coefficient of correlation ( $r$ value) between the scores of OSI and various measures of job attitudes and job behaviour. Highly significant positive correlation was found between the scores on the OSI and the scores on measures of such job related attitudinal and motivational and personality variables. 
Table 1. Occupational stressors and their indices.

\begin{tabular}{|l|l|l|}
\hline \multicolumn{1}{|c|}{$\begin{array}{c}\text { Sub-scales } \\
\text { (occupational stressors) }\end{array}$} & $\begin{array}{c}\text { Serial number of the } \\
\text { items in the schedule }\end{array}$ & $\begin{array}{c}\text { Range of } \\
\text { rabis }\end{array}$ \\
\hline Role Overload & $1,13,25,36,44,46$ & $.30-.46$ \\
\hline Role ambiguity & $2,14^{*}, 26,37$ & $.20-.48$ \\
\hline Role Conflict & $3,15^{*}, 27,38^{*}, 45$ & $.36-.53$ \\
\hline $\begin{array}{l}\text { Unreasonable group \& } \\
\text { Political pressures }\end{array}$ & $4,16,28,39$ & $.21-.52$ \\
\hline $\begin{array}{l}\text { Responsibility for } \\
\text { persons }\end{array}$ & $5,17,29$ & $.30-.57$ \\
\hline Under participation & $6^{*}, 18^{*}, 30^{*}, 40^{*}$ & $.55-.73$ \\
\hline Powerlessness & $7^{*}, 19^{*}, 31^{*}$ & $.44-.62$ \\
\hline Poor peer Relations & $8^{*}, 20,32^{*}, 41^{*}$ & $.24-.49$ \\
\hline Intrinsic impoverishment & $9,21^{*}, 33^{*}, 42$ & $.32-.64$ \\
\hline Low status & $10^{*}, 22^{*}, 34$ & $.48-.63$ \\
\hline Strenuous working \\
conditions
\end{tabular}

Depending on the 'true-keyed' or 'false-keyed' nature of the items, the following two different patterns of scoring were adopted:

\begin{tabular}{|l|c|c|}
\hline \multirow{2}{*}{ Categories of response } & \multicolumn{2}{|c|}{ Scores } \\
\cline { 2 - 3 } & $\begin{array}{c}\text { 'True-keyed' } \\
\text { items }\end{array}$ & $\begin{array}{c}\text { 'False-keyed' } \\
\text { items }\end{array}$ \\
\hline Never/strongly disagree & 1 & 5 \\
\hline Seldom/disagree & 2 & 4 \\
\hline Sometimes/undecided & 3 & 3 \\
\hline Mostly/agree & 4 & 2 \\
\hline Always/strongly agree & 5 & 1 \\
\hline
\end{tabular}

For ascertaining the validity and reliability of the new Bengali version (Appendix 1), 70 employees of different cadres ware randomly selected from two industries and two non productive organizations of Rajshahi City Corporation. In order to have a comparison of the original and adapted form of the test, 30 educated employees were selected from the same industries.

The procedure used in the study involved three steps. First, the English version of the scale was translated into Bengali and was checked and rechecked by the authors.
Second, to ascertain the appropriateness of the translation, four judges (two from the Department of English and two from the Department of Bengali, RU), were requested to examine carefully whether each item of both Bengali and English version conveys the same idea. Final form of the Bengali version was obtained by incorporating the suggestions offered by the judges. Then the Bengali version of the scale was supplied to three judges (teachers of the Psychology Department, RU) with a request to detect the items that appeared to be inappropriate for our culture. But the judges detected no such items. Third, in order to determine the testretest reliability (Annestasy and Susana 1997) of the newly constructed Bengali version of the test, it was administered to the subjects following standard procedure on two occasions at an interval of one month. The consistency between two sets of scores was computed by product moment correlation method (Garrett 1966). Finally, the original English version and the translated Bengali version of the scale were both administered on the same subjects at an interval of one month and co-efficient of correlation between the two sets of scores were computed.

The correlation co-efficient $(r=0.87)$ computed for assessing the test-retest reliability of the adapted OSI scale was highly significant $(\mathrm{t}=14.88$; $\mathrm{P}<0.01)$. Likewise the correlation co-efficient computed for testing the resemblance between the original and adapted version ( $\mathrm{r}=0.82 ; \mathrm{N}=30$ ) was also found significant $(\mathrm{t}$ $=4.36 ; \mathrm{P}<0.01)$. Since the main purpose of the study was to adapt an English version of OSI in Bengali in order to make it suitable for our culture, the results of the present investigation reveal that the translated version is worth using for such studies in Bangladesh.

\section{References}

Annestasy A and Susana U 1997. Psychological Testing. Persona Education. 105 pp.

Srivastava AK and Singh AP 1984. Manual of Occupational Stress Index. Manovaigyanik Parikchhan Sansthan, Varanasi, India.

Garrett HE and Woodworth RS 1966. Statistics in Psychology and Education. Longman Group Limited, London. 134 pp. 


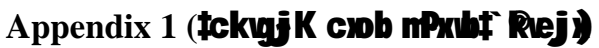

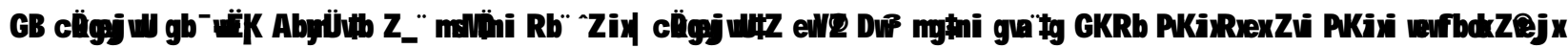

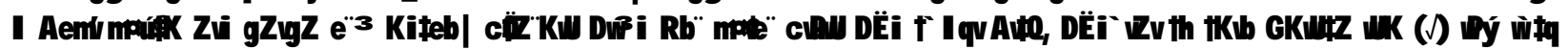

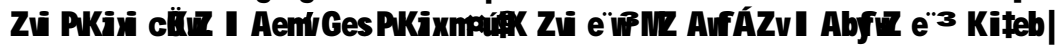

\begin{tabular}{|c|c|c|c|c|c|}
\hline 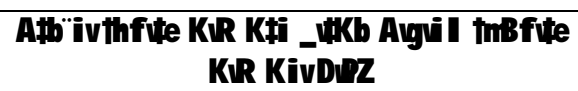 & 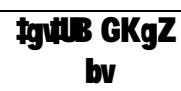 & $G K g Z$ bv & 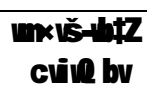 & GKgZ & maú GK gZ \\
\hline
\end{tabular}

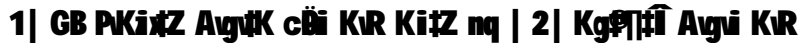

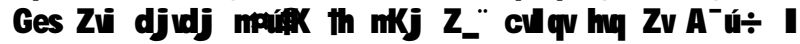

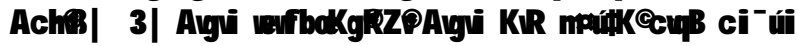

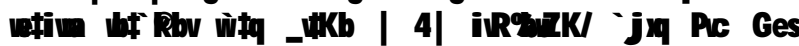

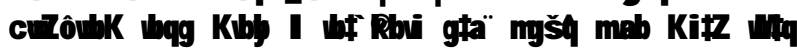

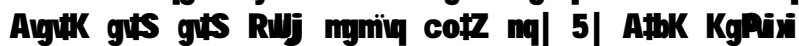

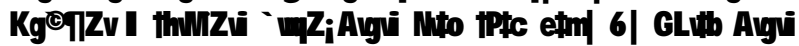

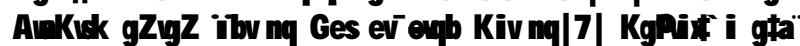

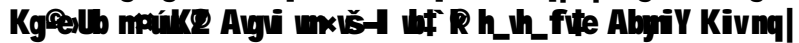

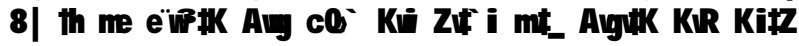

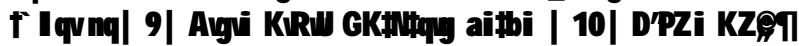

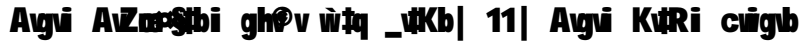

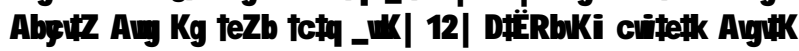

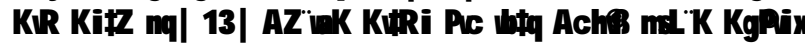

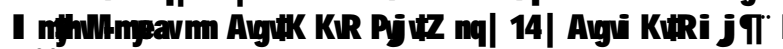

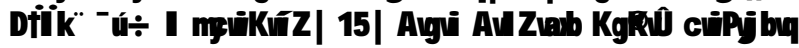

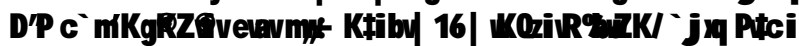

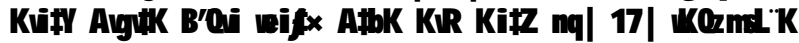

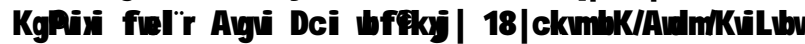

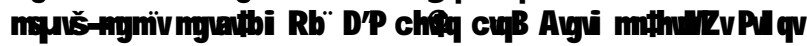

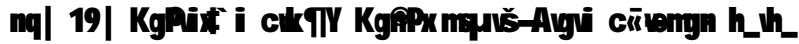
- i 'Zitc fa_

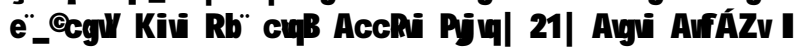

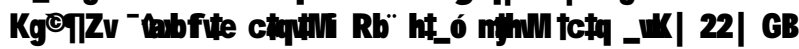

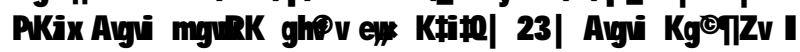

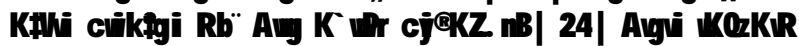

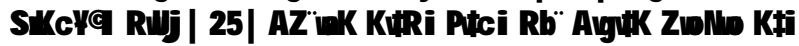

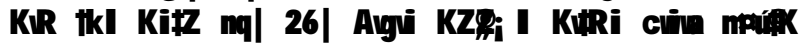

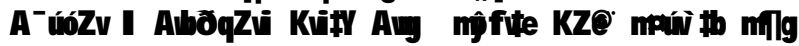

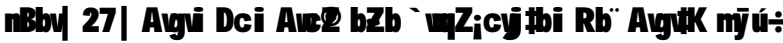

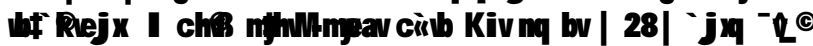

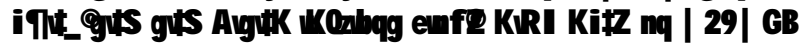

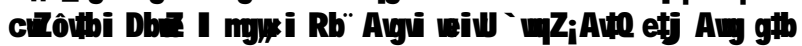

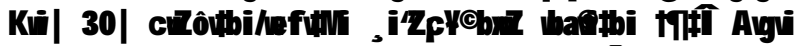

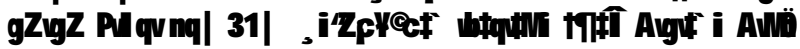

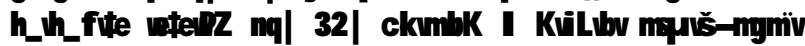

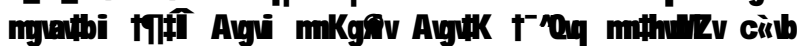

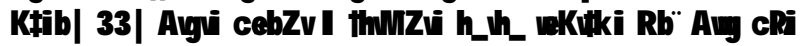

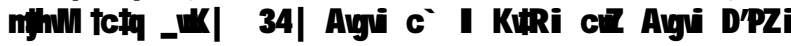

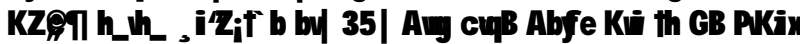

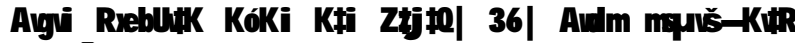

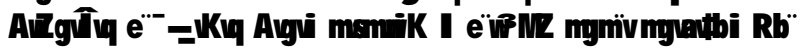

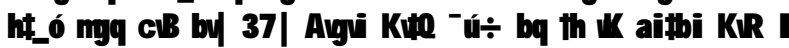

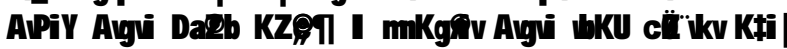

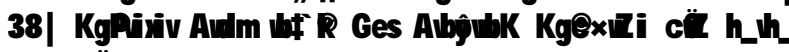

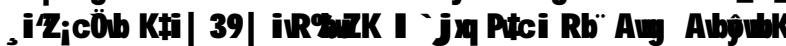

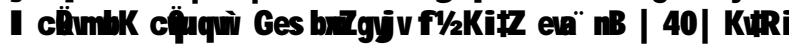
avi v, hš \& wZ GeskZ

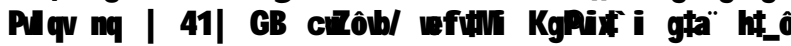

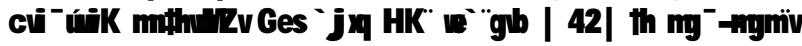

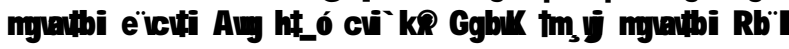

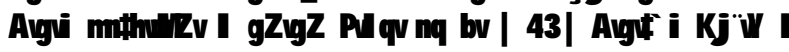
mothM myeavi wiK t_tK vetePbv Kifj GLutb KutR i cwitek

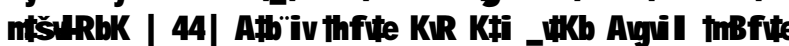

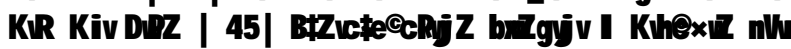

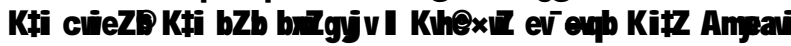

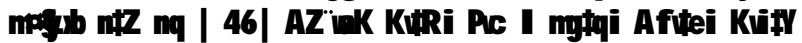

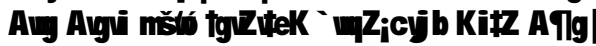

